\title{
INTERPRETIVE SUMMARIES, AUGUST 2013
}

Differences in particle characteristics and oxidized flavor as affected by heat-related processes of milk powder. By Li et al., page 4784. Stored milk powder has the problem of oxidized flavor, which affects consumers' sensory acceptability. We studied the effects of heat-related processes on particle characteristics and oxidized flavor of milk powder. The processes of preheat, concentration, and drying could accelerate the formation of oxidized volatiles in fresh and stored milk powder. Addition of ascorbic acid to milk powder changed the type of radical and reduced oxidation offflavor. In conclusion, the morphological characteristics of milk powder particles, not the surface composition, should be considered in maintaining the stability of oxidized flavor in storage.

http://dx.doi.org/10.3168/jds.2012-5799.

Effect of gum tragacanth on rheological and physical properties of a flavored milk drink made with date syrup. By Keshtkaran et al., page 4794. Date milk beverage as a nutritional flavored milk was formulated with gum tragacanth to obtain an acceptable mouthfeel. The samples contained 3 concentrations of 2 types of gum tragacanth, and particle size distribution, colorimetric assays, and sensory analysis were studied. Viscoelastic properties, flow behavior parameters, particle size, and color parameters were significantly affected by concentration of gum tragacanth, and the strength of this effect was influenced by the type of gum.

http://dx.doi.org/10.3168/jds.2012-5942.

Physical properties of pizza Mozzarella cheese manufactured under different cheese-making conditions. By Banville et al., page 4804. Two critical physical properties of pizza Mozzarella cheese - shreddability and meltability - were measured in experimental cheeses made under varying manufacturing conditions (addition of denatured whey proteins to milk, renneting $\mathrm{pH}$ of milk, aging of cheese, and temperature of experimentation). Cheese shreddability generally decreased with increasing shredding temperature and cheese age. The production of fines during shredding was reduced by decreasing the renneting $\mathrm{pH}$ of milk under controlled cheese-making conditions. It was possible to achieve desirable physical properties of Mozzarella during melting through careful setting of the cheesemaking conditions.

http://dx.doi.org/10.3168/jds.2012-6314.

Proteolysis and biogenic amine buildup in highpressure-treated ovine milk blue-veined cheese. By Calzada et al., page 4816. Blue-veined cheese made from ovine milk was pressurized at 400 or $600 \mathrm{MPa}$ after 3,6 , and 9 wk of ripening to extend product shelf- life by preventing overripening and buildup of biogenic amines (compounds of public health significance). Highpressure treatments of cheeses at 400 or $600 \mathrm{MPa}$ after 3 wk were the most effective in inactivating bacterial aminopeptidases, reducing free amino acid formation, and stabilizing flavor intensity. Biogenic amine buildup was influenced by pressurization. Tyramine concentration was lower in pressurized cheeses, but some of the pressurized cheeses showed higher tryptamine, phenylethylamine, or putrescine contents compared with control cheese.

http://dx.doi.org/10.3168/jds.2012-6409.

The occurrence of noncoagulating milk and the association of bovine milk coagulation properties with genetic variants of the caseins in 3 Scandinavian dairy breeds. By Poulsen et al., page 4830. Milk coagulation traits were examined in Danish Holstein (DH), Danish Jersey (DJ), and Swedish Red (SR) cows. The 3 breeds showed notable interbreed differences in milk coagulation properties, with DJ cows exhibiting superior milk coagulation capacity. In addition, milk from $2 \%$ of DH and $16 \%$ of SR cows was classified as noncoagulating. Furthermore, genetic variants of 3 casein genes were significantly associated with curd firming rate and rennet coagulation time. Our results indicated that selective breeding for variants associated with superior milk coagulation could increase raw milk quality and cheese yield in the 3 Scandinavian breeds. http://dx.doi.org/10.3168/jds.2012-6422.

Newly identified mutations at the CSN1S1 gene in Ethiopian goats affect casein content and coagulation properties of their milks. By Mestawet et al., page 485\%. New mutations were detected in exons and noncoding regions of the $\alpha_{S 1}$-casein gene (CSN1S1) in 4 different goat breeds in Ethiopia. Milk from animals carrying the new mutation had some increased milk components, such as protein content, and good cheesemaking properties. This finding will benefit breed selection for the emerging goat cheese industry. Farmers raising these goat breeds will also benefit from the increased selling price of goats. The finding has opened new interest in the susceptibility of protein in milk from animals with the new mutation to hydrolysis by digestive enzymes.

http://dx.doi.org/10.3168/jds.2012-6467.

A new method for production of low-fat Cheddar cheese. By Amelia et al., page 4870. A low-fat Cheddar cheese (LFCC) was produced by a process that used aged full-fat Cheddar cheese (FFCC) as an ingredient in combination with micellar casein concentrate, water, rennet, lactic acid, and salt. The texture of the LFCC was more similar to FFCC than to commercial 
reduced-fat Cheddar cheese. Flavor in all reduced-fat cheeses was less than that in FFCC, but the addition of commercial flavor concentrates to the LFCC might partially overcome this limitation. http://dx.doi.org/10.3168/jds.2012-6542.

Identification of milk proteins enhancing the antimicrobial activity of lactoferrin and lactoferricin. By Murata et al., page 4891. The antimicrobial activity of lactoferrin has been studied in detail; however, its cooperative action with other milk proteins remains to be elucidated. In this study, we identified milk proteins around $15 \mathrm{kDa}$ (bovine RNase 5, RNase 4 , and angiogenin-2) that enhanced the antimicrobial activity of lactoferrin against gram-negative bacteria, gram-positive bacteria, and fungus. Lactoferrin and the identified milk proteins around $15 \mathrm{kDa}$ may act concomitantly as antimicrobial agents in milk. http://dx.doi.org/10.3168/jds.2013-6612.

The dual effects of Maillard reaction and enzymatic hydrolysis on the antioxidant activity of milk proteins. By Oh et al., page 4899. The Maillard reaction (nonenzymatic browning) is a complex reaction that occurs between carbonyl and amine groups during food processing and storage. We investigated the synergistic effects of Maillard reaction and hydrolysis on the antioxidant activity of milk protein containing whey protein concentrate and sodium caseinate. The Maillard reaction coupled with hydrolysis procedures showed significantly greater antioxidant activity than did either hydrolysis or Maillard reaction alone. The greatest antioxidant properties were observed in whey MRP hydrolyzed by the commercial protease Alcalase. A combination of Maillard reaction and hydrolysis could provide new opportunities for food-grade antioxidants in the pharmaceutical, food, and dairy industries. http://dx.doi.org/10.3168/jds.2013-6613.

Antimicrobial activity of binary combinations of natural and synthetic phenolic antioxidants against Enterococcus faecalis. By Gutiérrez-Fernández et al., page 4912. The phenolic compounds thymol, carvacrol, gallic acid, butylated hydroxyanisole, and octyl gallate, individually tested, exhibited antimicrobial activity against 4 dairy isolates of Enterococcus faecalis with different virulence factors. The most effective binary combinations of these phenolic compounds were thymol-carvacrol and gallic acid-octyl gallate. These results highlight the possibility of using combinations of phenolic compounds to inhibit the growth of potential virulent or spoilage E. faecalis strains by reducing the total amount of additives used in dairy foods.

http://dx.doi.org/10.3168/jds.2013-6643.

Irradiation dose detection of irradiated milk powder using visible and near-infrared spec- troscopy and chemometrics. By Kong et al., page 4921. A fast detection method for irradiation dose of irradiated milk powder was investigated using visible/ near-infrared spectroscopy and chemometrics. Different chemometric methods were compared to determine the optimal prediction performance. The best result was achieved by using an extreme learning machine model with correlation coefficients of 0.97 . This study described a new approach for the fast detection of irradiation dose of milk powder. The results would be helpful for quality detection and safety monitoring of milk powder

http://dx.doi.org/10.3168/jds.2013-6659.

The microbial content of raw and pasteurized cow milk as determined by molecular approaches. By Quigley et al., page 4928. Milk can contain a variety of bacteria that play a role in the subsequent fermentation of milk and affect the shelf-life and food safety of milk and milk products. Milk is pasteurized to reduce the bacterial load present, thereby removing pathogenic bacteria and extending shelf-life. We investigated the identity of the bacteria present in milk before and after pasteurization using state-of-the-art DNAbased approaches. Our study revealed the presence of diverse bacterial communities in milk and indicated, for the first time, the presence of a number of bacteria not previously detected in milk.

http://dx.doi.org/10.3168/jds.2013-6688.

Explaining tolerance for bitterness in chocolate ice cream using solid chocolate preferences. $B y$ Harwood et al., page 4938. Chocolate ice cream is commonly formulated with more sugar than nonchocolate flavors to compensate for inherent bitterness, which is an integral part of the complex flavor of chocolate. However, in light of the worldwide obesity epidemic, sugar levels in food are of great concern for many consumers. The main goal of this study was to estimate rejection thresholds for bitterness in chocolate ice cream in the context of market segments that prefer milk chocolate or dark chocolate. Estimating rejection thresholds could be an effective strategy for determining acceptable formulations when considering attributes that become objectionable at high intensity.

http://dx.doi.org/10.3168/jds.2013-6715.

The biodiversity of lactococcal bacteriophages isolated from 3 Gouda-type cheese-producing plants. By Murphy et al., page 4945. Lactococcus lactis, one of the most intensely utilized starters globally, carries significant industrial and economic importance. This study identified 20 distinctly different phages from whey samples obtained from 3 cheese factories that used 2 undefined starter cultures. The data revealed that these phages were all 936-type phages. Our results highlight the ubiquity, prevalence, and diversity of 
these bacterial parasites and the continued threat they pose to a modern dairy fermentation plant.

http://dx.doi.org/10.3168/jds.2013-6748.

Immunomodulatory and hypoallergenic properties of milk protein hydrolysates in ICR mice. By Pan et al., page 4958. Milk protein is usually the first source of foreign antigens that infants ingest in large quantities. Because their intestinal systems are insufficiently developed to digest and immunologically react to milk proteins, infants and children are considered members of a high-risk group of individuals. Various milk proteins and their enzymatically derived peptides have demonstrated modulating effects in vitro, including lymphocyte activation and proliferation. We established in vivo that in ovalbumin-sensitized ICR mice, milk protein hydrolysates made from full-cream milk exhibited immunomodulatory and hypoallergenic properties.

http://dx.doi.org/10.3168/jds.2013-6758.

Herd-level relationship between antimicrobial use and presence or absence of antimicrobial resistance in gram-negative bovine mastitis pathogens on Canadian dairy farms. By Saini et al., page 4965. Emergence of antimicrobial resistance has serious consequences for human and animal health. Surveillance of antimicrobial use and resistance is therefore needed for containing antimicrobial resistance in bacteria. The present study examined an association between herd-level antimicrobial use and resistance in bovine mastitis Escherichia coli $(\mathrm{n}=394)$ and Klebsiella species isolates $(\mathrm{n}=139)$ on 89 Canadian dairy farms. The presence of ampicillin-intermediate or ampicillinresistant E. coli isolates was associated with the use of intramammary administered cloxacillin, penicillinnovobiocin combination, and cephapirin used for dry cow therapy, intramammary ceftiofur administered for lactating cow therapy, and systemically administered penicillin. No associations were observed for Klebsiella species isolates.

http://dx.doi.org/10.3168/jds.2012-5713.

Milk lactoferrin in heifers: Influence of health status and stage of lactation. By Chaneton et al., page $497 \%$. Bovine mastitis remains a major concern for the dairy industry. The mammary gland displays a wide range of mechanisms to prevent and combat bacterial infections. Lactoferrin, a glycoprotein present in milk, is one of those mechanisms. In this study, we presented evidence that the role of lactoferrin is to respond to infections, rather than to prevent infections, particularly during the first weeks of lactation when other immunologic elements of the mammary gland are impeded. Additionally, we demonstrated that lactoferrin concentration in early lactation may be used to pre- dict the risk of future infections in individual mammary quarters.

http://dx.doi.org/10.3168/jds.2012-6028.

Efficacy of extended cefquinome treatment of clinical Staphylococcus aureus mastitis. By Swinkels et al., page 4983. Staphylococcus aureus is an important clinical mastitis-causing pathogen that is difficult to cure. Extended intramammary treatment for mastitis is frequently used in the expectation of a better outcome. To evaluate the efficacy of such treatment regimens, extended treatment was compared with standard treatment on farms in 5 European countries. Clinical cure, but not bacteriological cure, was significantly better after extended treatment. Extending treatment of clinical Staph. aureus mastitis is not recommended.

http://dx.doi.org/10.3168/jds.2012-6197.

The effect of repeated episodes of bacteria-specific clinical mastitis on mortality and culling in Holstein dairy cows. By Cha et al., page 4993. The objective of this study was to estimate the effects of first and repeated cases of bacteria-specific clinical mastitis on the risk of mortality and culling in Holstein dairy cows. The pathogens studied were Streptococcus spp., Staphylococcus aureus, Staphylococcus spp., Escherichia coli, Klebsiella spp., Trueperella pyogenes, and others, as well as no growth on aerobic culture. The results from this study can guide farmers in identifying which pathogens require particular attention to reduce cow exit. The mortality risks from this study will be used to parameterize an economic model to provide dairy farmers economically optimal decisions for their diseased cows.

http://dx.doi.org/10.3168/jds.2012-6232.

Liver fat content and lipid metabolism in dairy cows during early lactation and during a midlactation feed restriction. By Gross et al., page 5008. Effects of negative energy balance (NEB) in early and mid lactation on hepatic lipidosis and lipid metabolism were studied in dairy cows. In contrast to NEB during early lactation, a similar NEB induced by feed restriction during mid lactation did not cause the development of fatty liver. Differences of liver lipid metabolism between the experimental periods were also reflected by differences of messenger RNA abundance of key factors in the liver tissue.

http://dx.doi.org/10.3168/jds.2012-6245.

Perceptions and risk factors for lameness on organic and small conventional dairy farms. $B y$ Richert et al., page 5018. The aim of this study was to characterize farmers' perceptions of lameness and identify risk factors for prevalence of lameness on organic 
and similarly sized conventional farms. On the relatively small, lower-producing farms included in this study, the prevalence of lameness scored by study personnel was less than previously reported for larger, higherproducing dairy herds located in the United States. Prevalence of lameness and rate of farmer-identified lameness events demonstrated a weak positive correlation. Risk factors for lameness prevalence included body condition, hock lesions, use of a footbath, and length of time cows spent outdoors.

http://dx.doi.org/10.3168/jds.2012-6257.

Hazard perception of Dutch farmers and veterinarians related to dairy young stock rearing. $B y$ Boersema et al., page 502\%. A total of 110 dairy farmers and 26 bovine veterinarians participated in a web-based questionnaire that transferred their perception regarding several hazards into a utility score to subsequently rank the outcome. The survey was divided over 6 periods of a young stock rearing process. For most periods, the ranking of the hazard perceptions differed between veterinarians and farmers. In only one period (period IV; pregnancy period until 4 wk before calving) did farmers and veterinarians have the same top 3 ranking of the hazards "mastitis," "abortion," and "poor growth rate." The outcome of this study demonstrated the difference in hazard perception of veterinarians and farmers, which can be used to improve communication and consultation in the calf-rearing process on dairy farms. http://dx.doi.org/10.3168/jds.2012-6276.

Comparison of updates to the Molly cow model to predict methane production from dairy cows fed pasture. By Gregorini et al., page 5046. Cumulative improvements to Molly have not been validated for $\mathrm{CH}_{4}$ production under pasture-based diets. The predictions of enteric methane from 2 updated versions of Molly were compared using 2 different ruminal volatile faty acid stoichiometry settings. The model was challenged with data from dairy cows fed fresh-cut grass in respiration chambers. Updates improved the precision of methane prediction, demonstrating the utility of improvements in Molly to formulate research questions better and compare methane mitigation strategies. http://dx.doi.org/10.3168/jds.2012-6288.

Evaluation of an alternative dosing regimen of a J-5 mastitis vaccine against intramammary Escherichia coli challenge in nonlactating lategestation dairy cows. By Gurjar et al., page 5053. An alternative vaccination scheme for a J-5 bacterin was evaluated in a novel late-gestation Escherichia coli intramammary infection (IMI) challenge model. Vaccination resulted in significantly increased serum immunoglobulin G ( $\operatorname{IgG}$ ) titers, particularly of the IgG1 isotype. Intramammary challenge resulted in IMI establishment in $85 \%$ of quarters. Vaccination did not prevent new IMI but generally reduced clinical severity of resulting clinical mastitis cases.

http://dx.doi.org/10.3168/jds.2012-6456.

Gradual cessation of milking reduces milk leakage and motivation to be milked in dairy cows at dry-off. By Zobel et al., page 5064. Milk leakage and behavior were monitored in 24 high-production cows dried off using 2 methods: gradual and abrupt dry-off. Cows dried off by gradual cessation of milking showed no increase in standing at the gate in anticipation of milking, whereas the odds of abruptly dried-off cows standing at the gate were 5 times as great. Milk leakage was more prevalent in the abruptly dried-off cows and these cows leaked for a longer period. Because milk leakage has been linked to an increase in intramammary infections in the next lactation, this study indicates that high-producing cows benefit from gradual reduction in milking at dry-off.

http://dx.doi.org/10.3168/jds.2012-6501.

Influence of breed, milk production, season, and ambient temperature on dairy cow reticulorumen temperature. By Liang et al., page 5072. Automatic monitoring of core body temperature in dairy cattle could be useful for identification of illness, heat stress, general physiological stress, and estrus. In this study, using an automatic reticulorumen temperature recording device (SmartBolus, TenXSys Inc., Eagle, ID), ambient temperature, breed, milk yield, season, and time of day influenced dairy cow reticulorumen temperature. Results of this research provide new understanding of the relationships among environmental factors, cow factors, and body temperature.

http://dx.doi.org/10.3168/jds.2012-6537.

Rumination time during the summer season and its relationships with metabolic conditions and milk production. By Soriani et al., page 5082. This study reports the effects of hot conditions during summer on rumination time and its relationship with metabolic conditions and milk production. Hot conditions negatively affected rumination time and modified rumination behavior during daytime and nighttime. The reduction of rumination time was related to the behavior of physiological markers of heat stress and milk yield reduction. The measurement of rumination time during the summer season could be used in a system to counteract the negative effect of hot season on animal welfare and milk yield.

http://dx.doi.org/10.3168/jds.2013-6620.

The effect of intramammary infection with coagulase-negative staphylococci in early lactating heifers on milk yield throughout first lactation revisited. By Piepers et al., page 5095. This study scrutinized further the somewhat surprising 
positive association between intramammary infection with coagulase-negative staphylococci (CNS) in early lactating heifers and test-day milk yield throughout first lactation. Data from 240 heifers from 29 herds were used. With appropriate correction for confounders and a number of biologically understood intermediate variables in the model, heifers infected with CNS in early lactation produced $2.0 \mathrm{~kg} / \mathrm{d}$ more throughout first lactation than did noninfected herdmates.

http://dx.doi.org/10.3168/jds.2013-6644.

CD2/CD21 index: A new marker to evaluate udder health in dairy cows. By Schwarz et al., page 5106. Mastitis diagnosis in dairy cows is based on somatic cell counts and bacteriological examinations of quarter foremilk samples. Studies differentiating the responsible cell populations in milk samples of healthy udder quarters revealed lymphocytes as the dominant cell population. To conduct a detailed investigation of lymphocyte subpopulations, we analyzed $\mathrm{CD} 2^{+} \mathrm{T}$ and $\mathrm{CD} 21^{+} \mathrm{B}$ lymphocytes in quarter foremilk samples and found an opposite trend of percentages of these populations in healthy and diseased udder quarters, resulting in the definition of a new CD2/CD21 index. Our results indicated a connection between this index and current or previous presence of mastitis pathogens.

http://dx.doi.org/10.3168/jds.2013-6804.

Glomerular filtration rate in Holstein dairy cows estimated from a single blood sample using iodixanol. By Murayama et al., page 5120. This study established a simplified procedure for estimating glomerular filtration rate (GFR) in Holstein dairy cows by using a single blood sample and the contrast medium iodixanol as a test substance. This reduces the handling-associated stress and possible pain caused by multisampling strategies. The simplified procedure involving the estimated volume of distribution is a practical, ethical alternative for estimation of GFR in dairy cows.

http://dx.doi.org/10.3168/jds.2013-6884.

Strain-specific pathogenicity of putative hostadapted and nonadapted strains of Streptococcus uberis in dairy cattle. By Tassi et al., page 5129. Streptococcus uberis is a common cause of clinical and subclinical mastitis in dairy cattle. Putatively host-adapted and nonadapted strains of Strep. uberis have been identified based on epidemiological data. Here, we described the host response to challenge with putatively host-adapted and nonadapted Strep. uberis and showed that the strains differed in pathogenicity based on clinical, bacteriological, and immunological responses. This suggests that Strep. uberis is not merely an opportunistic environmental pathogen. We also provide preliminary evidence that interleukin (IL)-17A, a cytokine involved in control of extracellular bacterial infections, may be involved in the resolution of intramammary infection in dairy cows.

http://dx.doi.org/10.3168/jds.2013-6741.

Short communication: Circulating serotonin (5HT) concentrations on day 1 of lactation as a potential predictor of transition-related disorders. By Laporta et al., page 5146. Circulating serum concentrations of the monoamine serotonin $(5-\mathrm{HT})$ on d 1 of lactation in Holstein cows were negatively correlated with milk fever incidence (d 1 postpartum) and ketosis grade (most severe ketosis incidence recorded during the first $10 \mathrm{~d}$ postpartum). Additionally, serum 5 -HT on d 1 of lactation was positively correlated with concentrations of circulating serum ionized calcium and plasma parathyroid hormone-related protein on d 1 of lactation. Increased circulating concentrations of 5-HT might decrease milk fever events at the onset of lactation and ketosis severity during the first $10 \mathrm{~d}$ postpartum in dairy cows.

http://dx.doi.org/10.3168/jds.2013-6718.

Ruminal degradability of dry matter, crude protein, and amino acids in soybean meal, canola meal, corn, and wheat dried distillers grains. By Maxin et al., page 5151. Adequate estimation of rumen disappearance of feed protein and amino acids (AA) is essential for correct determination of metabolizable protein and AA digestive flow with the current systems used to balance dairy rations. This study assessed, using the in situ technique, dry matter, crude protein, and AA ruminal degradation of soybean meal and 3 others protein supplements (canola meal, high protein corn, or wheat dried distillers grains) frequently used in North American dairy rations to replace soybean meal. http://dx.doi.org/10.3168/jds.2012-6392.

Milk composition and flavor under different feeding systems: A survey of dairy farms. By Yayota et al., page 5174. Understanding the causes of regional milk flavors will help a targeted marketing strategy and conserve regional feed resources. This study evaluated the influence of regional differences in feeding systems on milk composition and flavor at the farm level. We investigated dairy farms that used grass silage, maize silage, or by-products as characteristic feed components according to regional feed resources. The results indicated that the differences in the feeding systems contribute to the observed differences in the fatty acid compositions of the milks; however, more exclusive feeding systems would be required to provide a unique regional flavor to the milk.

http://dx.doi.org/10.3168/jds.2012-5963.

Chewing activities and particle size of rumen digesta and feces of precision-fed dairy heifers fed different forage levels with increasing levels 
of distillers grains. By Suarez-Mena et al., page 5184. Effects of forage concentration and corn dry distillers grains with solubles (DDGS) level were evaluated in precision-fed dairy heifer diets. Chewing time increased at higher forage concentration and with the addition of DDGS. Forage content increased eating time, whereas DDGS level increased ruminating time. Higher forage increased rumen digesta weight and volume. Fecal particle size was not influenced by forage level but increased with DDGS level, whereas particle size of rumen contents was greater at higher forage content. Forage and DDGS levels did not affect rumen $\mathrm{pH}$. When used in dairy heifer rations as a replacement for canola meal, DDGS yielded similar rumen digestion traits and will likely promote similar production results. http://dx.doi.org/10.3168/jds.2012-6155.

The effect of timing of oral meloxicam administration on physiological responses in calves after cautery dehorning with local anesthesia. By Allen et al., page 5194. Welfare concerns regarding painful routine animal husbandry procedures are increasing. Parenteral meloxicam is an efficacious analgesic for use before dehorning in calves. This study compared the effects of oral meloxicam when administered $12 \mathrm{~h}$ before or at the time of dehorning.

http://dx.doi.org/10.3168/jds.2012-6251.

Aerobic deterioration stimulates outgrowth of spore-forming Paenibacillus in corn silage stored under oxygen-barrier or polyethylene films. $B y$ Borreani et al., page 5206. Psychrotolerant Paenibacillus spp. spores appear to be the most limiting factor in extending the shelf-life of pasteurized milk. Silages could be a source of contamination of milk at the farm level. Aerobic exposure of corn silage increased the number of Paenibacillus spores to levels that could contaminate the diets of dairy cows. Any effort to reduce aerobic deterioration, such as the use of oxygen-barrier films to cover silages, could help reduce the potential for Paenibacillus spore contamination within dairy rations and, consequently, the risk of milk contamination. http://dx.doi.org/10.3168/jds.2013-6649.

What do preweaned and weaned calves need in the diet: A high fiber content or a forage source? By Terré et al., page 521\%. The inclusion of forage in the diet of young calves is controversial. This study indicates that including a forage source, rather than a high-fiber concentrate, in the diet of young calves, especially around weaning, is a good strategy to increase rumen $\mathrm{pH}$, stimulate starter intake, and therefore improve weight gain. Furthermore, having a forage source available stimulates rumination and reduces abnormal behaviors.

http://dx.doi.org/10.3168/jds.2012-6304.
Effects of forage provision to young calves on rumen fermentation and development of the gastrointestinal tract. By Castells et al., page 5226. Providing poor-quality chopped forage to young calves has been demonstrated to improve growth performance. The rumens of calves that had access to forage, either alfalfa or oat hay, presented a greater $\mathrm{pH}$ of the rumen liquid and an increased expression of a gene involved in volatile fatty acid absorption in the rumen wall. Therefore, because of the better rumen environment, these calves consumed a greater amount of solid feed, resulting in improved performance compared with calves deprived of forage. In calves receiving oat hay, the increased live body weight gain was due to a carcass, rather than a gastrointestinal tract, weight gain. http://dx.doi.org/10.3168/jds.2012-6419.

Corn silage in dairy cow diets to reduce ruminal methanogenesis: Effects on rumen metabolically active microbial communities. By Lettat et al., page 5237. The objective of this study was to assess the effects of replacing alfalfa silage with corn silage in dairy cow diets on the metabolically active ruminal microbial communities (bacteria, methanogens, and protozoa) using molecular biology techniques [complementary (c) DNA-based length heterogeneity (LH)-PCR and quantitative PCR]. The cDNA-based LH-PCR revealed important differences in bacterial communities, whereas quantitative PCR showed an increase in the number and activity of methanogens despite lower methane energy losses induced by the dietary shift in forage source from alfalfa silage to corn silage. This suggests that the use of cDNA-based quantitative PCR to estimate archaeal growth and activity is not sufficiently reliable to reflect changes in ruminal methanogenesis. Consequently, more reliable molecular biology tools are required to better characterize changes in methanogen community structure in order to enhance our understanding of the microbial process involved in ruminal methanogenesis and hence, enable the development of more effective dietary methane-mitigation strategies.

http://dx.doi.org/10.3168/jds.2012-6481.

Effect of feeding according to energy balance on performance, nutrient excretion, and feeding behavior of early lactation dairy cows. By Maltz et al., page 5249. Cows were assigned to a control or precision diet that adjusted the dietary caloric density weekly based on energy balance by manipulating the additional concentrate fed from 0 to $25 \%$ of the diet. Feeding a precision diet improved yields of milk and milk components, and feed conversion ratio; however, nutrient digestibility and $\mathrm{N}$ excretion did not change. Measures of energy status were mildly affected, but precision cows selected a diet in favor of longer neutral detergent fiber particles and spent more time ruminat- 
ing lying. Feeding a diet according to the needs of cows improved production and feed efficiency.

http://dx.doi.org/10.3168/jds.2013-6549.

Effect of forage conservation method on plasma lipids, mammary lipogenesis, and milk fatty acid composition in lactating cows fed diets containing a 60:40 forage-to-concentrate ratio. $B y$ Halmemies-Beauchet-Filleau et al., page 526\%. The effects of forage conservation on plasma lipids, mammary lipogenesis, and milk fat composition were examined in 2 complementary experiments. Compared with zerograzing, hay increased milk fat 10:0 to 16:0 content and decreased cis-9 18:1, 18:2n-6, cis-9,trans-11 18:2, and 18:3n-3 concentrations. Conservation of grass by ensiling rather than drying altered milk fat secretion, but had little influence on plasma lipids, mammary lipid metabolism, or milk fat composition. Changes in milk fatty acid composition on fresh grass relative to conserved forages were associated with a lower calculated energy balance, higher uptake of preformed fatty acids, and decreased synthesis of 16:0 de novo in the mammary glands.

http://dx.doi.org/10.3168/jds.2013-6571.

Short communication: Evaluation of acid-insoluble ash and indigestible neutral detergent fiber as total-tract digestibility markers in dairy cows fed corn silage-based diets. By Lee and Hristov, page 5295. This study compared nutrient digestibility coefficients determined using 2 intrinsic markers, acidinsoluble ash and indigestible neutral detergent fiber, with total fecal collection data. Overall, indigestible neutral detergent fiber appeared to be a more reliable digestibility marker than acid-insoluble ash in terms of detecting dietary differences in apparent digestibility of some nutrients. Interactions with diet composition, however, need to be considered when estimating totaltract digestibility using intrinsic markers.

http://dx.doi.org/10.3168/jds.2012-6442.

Short communication: Supplementing lysine and methionine in a lactation diet containing a high concentration of wet corn gluten feed did not alter milk protein yield. By Mullins et al., page 5300. The effects of using 2 commercial supplements to supply additional metabolizable amino acids lysine and methionine were evaluated. Ninety-six lactating Holstein cows were assigned to 1 of 8 pens. Pens were offered rations formulated to differ in metabolizable amino acid supply. Feed intake and production were monitored daily, and milk components were analyzed 3 d/wk for 4 wk. No effects of supplemental amino acids were observed for intake and production. http://dx.doi.org/10.3168/jds.2013-6755.
Genomic selection using indicator traits to reduce the environmental impact of milk production. By Hansen Axelsson et al., page 5306. Dairy cows emit a substantial amount of greenhouse gases into the atmosphere, and no direct breeding is conducted to reduce this environmental impact. The results from this simulation study showed that breeding goals with production and functional traits are beneficial for the environment, but the genetic gain in reducing environmental impact can be increased by almost 35\% if an indicator trait such as methane measured in the breath of the cow during milking is used in the genetic evaluation.

http://dx.doi.org/10.3168/jds.2012-6041.

Accuracy of genomic prediction for milk production traits in Chinese Holstein population using a reference population consisting of cows. By Ding et al., page 5315. Progeny-tested bulls are used to form reference populations in almost all countries where genomic selection in dairy cattle has been implemented. In this study, we investigated the accuracy of genomic prediction when cows were used to form the reference population. The results showed that the realized accuracies (obtained from a validation population of progeny-tested bulls) and theoretical accuracies were 0.13 to 0.30 and 0.23 to 0.33 higher than the accuracies of conventional pedigree indices of young candidate bulls in China, respectively. The results indicate that, as an alternative, genomic selection using cows to form the reference population is feasible.

http://dx.doi.org/10.3168/jds.2012-6194.

Feasibility of using automatic milking system data from commercial herds for genetic analysis of milkability. By Carlström et al., page 5324. We studied whether it was possible to use data from automatic milking systems (AMS) in commercial herds to estimate genetic parameters for various milkability traits. We found high heritability estimates for flow rate and box time, indicating that it should be relatively easy to improve these traits by selection. However, only $47 \%$ of the data was useful because of insufficient identity recording and incomplete, or inconsistent, records. We concluded that AMS data are very useful for genetic analysis of milkability traits, although extensive data editing is necessary.

http://dx.doi.org/10.3168/jds.2012-6221.

Genomic architecture of bovine $\kappa$-casein and $\beta$-lactoglobulin. By Gambra et al., page 5333. A genome-wide association study was performed to identify single nucleotide polymorphisms associated with $\kappa$-casein and $\beta$-lactoglobulin concentrations in milk of crossbred Holstein $\times$ Jersey population using the 
BovineHD Beadchip (Illumina Inc., San Diego, CA). Sixty-one markers were found to be associated with milk composition traits. Subsequent pathway analysis revealed several pathways that have roles in $\kappa$-casein and $\beta$-lactoglobulin regulation through kinase and phosphatase activities.

http://dx.doi.org/10.3168/jds.2012-6324.

Genetic parameters for body condition score, locomotion, angularity, and production traits in Italian Holstein cattle. By Battagin et al., page 5344. In this study, the heritabilities of body condition score and locomotion, recently included in the national type traits recording system for the Italian Holstein breed, were estimated. Genetic and phenotypic correlations between body condition score and locomotion, and between them and angularity, milk yield, and milk contents were assessed. The additive genetic variance for body condition score and locomotion was low but comparable with previous findings. Before including body condition score and locomotion in the selection index, genetic relationships with other type and functional traits need to be investigated.

http://dx.doi.org/10.3168/jds.2012-6352.

Production and reproduction of Fleckvieh, Brown Swiss, and 2 strains of Holstein-Friesian cows in a pasture-based, seasonal-calving dairy system. By Piccand et al., page 5352. This study compared production and reproduction of Swiss with New Zealand Holstein-Friesian cows in a pasture-based, seasonal-calving dairy system. The Swiss Holstein-Friesian cows were not suited for this production system because of their poor reproductive outcomes, despite their high milk production efficiency. In contrast, the Swiss Fleckvieh cows fulfilled the compact-calving objectives of reproduction and should receive further attention despite their lower production outcomes per lactation. http://dx.doi.org/10.3168/jds.2012-6444.

The estimation of genomic relationships using breedwise allele frequencies among animals in multibreed populations. By Makgahlela et al., page 5364. Approaches for calculating genomic relationships are based on single-breed populations and commonly use allele frequencies (AF) of the genotyped animals.
The calculation of relationships in multibreed populations needs to be extended to allow for differences in AF between breeds. Methods based on estimated $\mathrm{AF}$ from the current genotypes gave generally higher estimated relationships. Methods that used AF from the base (founder) population were more precise and relatively comparable to traditional pedigree relationships, particularly when AF were estimated for the base population for each breed. This measure of genomic relationships could play a crucial role in genomic evaluations for multibreed populations.

http://dx.doi.org/10.3168/jds.2012-6523.

Application of a posteriori granddaughter and modified granddaughter designs to determine Holstein haplotype effects. By Weller et al., page 5376. A posteriori and modified granddaughter designs were applied to determine haplotype effects of genotyped Holstein bulls and cows. Of 33 traits analyzed with the a posteriori granddaughter design, the withinfamily haplotype effect was important for all but 2 traits. Of 21 traits analyzed with the modified granddaughter design, the within-family haplotype effect was important for 8 traits. Although the results can be used to determine causative mutations for most of the analyzed traits analyzed, complete DNA sequencing probably will be needed for most of the analyzed sires. http://dx.doi.org/10.3168/jds.2013-6650.

Angiopoietin-2 (ANGPT2) as a candidate gene for somatic cell score in German Holstein cattle. By Tetens et al., page 5388. The bovine angiopoietin-2 gene (ANGPT2) is located within a previously finemapped quantitative trait locus (QTL) region for somatic cell score. It is involved in the extravasation of neutrophils and is a promising candidate gene for this trait. Analyzing a granddaughter design, significant trait associations were found for polymorphisms in ANGPT2. Differential messenger RNA expression in the udder lymph nodes of animals presumably carrying divergent QTL alleles was demonstrated, indicating a role of ANGPT2 in cell score, and possibly implying the mechanism of neutrophil extravasation as an unfavorable target of selection. http://dx.doi.org/10.3168/jds.2013-6798. 